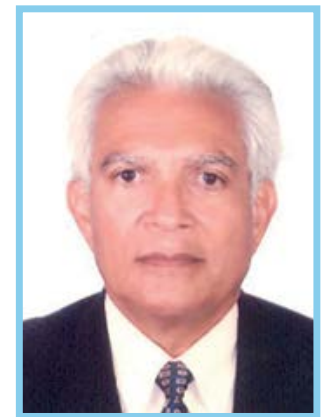

Agustín Campos Arenas, Ph. D.

\title{
LA EDUCACIÓN UNIVERSITARIA DE LA MUJER
}

\section{RESUMEN}

El presente artículo hace un recorrido sobre la educación universitaria de la mujer en diferentes países, incluyendo al Perú. Se muestra el desafío que ha significado que la mujer pueda acceder a la educación universitaria y las luchas que mujeres y hombres de avanzada tuvieron que realizar para que la educación universitaria sea también para las mujeres.

Se ha incluido nombres y fechas, como un elemento importante, en una historia de la educación de la mujer que podría interesar al lector.

\section{ABSTRACT}

The present article talks about the university education of women in different countries, including Peru. It shows the challenge that women are able to access to university education and the struggles that women and men had to perform for the university education are able for women.

It includes names and dates, as an important element in a story of woman education that could interest the reader.

\section{PALABRAS CLAVE}

Doctrina Social de la Iglesia, ética, moral, educación superior.

\section{KEYWORDS}

Social Doctrine of the Church, ethics, moral, higher education.
1 pesar de que en ciertos momentos en la historia de la humanidad se encuentran mujeres con importantes contribuciones en determinados campos del saber, tales como: Hypatia (filósofa griega, 350 - 415 a. c.) y Trótula, la médico italiana (1035 d. c.), puede afirmarse que la educación de la mujer a lo largo de los siglos ha sido negada, descuidada o poco desarrollada, según los países y los tiempos. La exclusión, discriminación o marginación de la mujer en educación ha respondido a cuestiones culturales arraigadas en sociedades dominadas por hombres. Esto, se ve claramente cuando se analizan las tasas de analfabetismo desagregadas de acuerdo a sexo; el porcentaje de analfabetismo de las mujeres es siempre más alto, especialmente, en zonas rurales y de pobreza.

Lasaccionesdecrianzadeloshijosyresponsabilidades delhogar son mencionados, también, comolimitantes de la educación de la mujer. De allí que en cursos de alfabetización y de capacitación técnica, las mujeres tienden a abandonarlos, a asistir irregularmente o perder la motivación de logro (UNESCO, 1997).

Por ello, desde hace más de 30 años se viene hablando en diferentes foros internacionales (e.g. Quinta Conferencia Internacional, Hamburgo, 1977) de la discriminación por género con relación a la educación 
de la mujer, con el objeto de eliminar prácticas discriminatorias y falta de equidad de género, en busca de justicia entre el hombre y la mujer. Se considera que la educación le permitirá participar más activamente en la sociedad y tener conocimientos y habilidades que le facilitarán su integración en una sociedad igualitaria.

La discriminación y marginación de la mujer, en cuanto a la educación, tiene implicancias posteriores en la consecución de un empleo digno y la obtención de salarios comparables con los del hombre. "Para el 2002, los ingresos que percibían las mujeres en el mercado de trabajo eran equivalentes al $68 \%$ de los obtenidos por los hombres con similar nivel educativo" (Ungo, 2007). Esto, implica desigualdad de oportunidades y de consideración en el campo laboral. Por ello, es fácil encontrar a mujeres desarrollando tareas laborales de bajo nivel remunerativo o actividades temporales.

La educación es un derecho humano fundamental para el desarrollo personal y social. En el caso de la mujer, es un requisito fundamental para su desarrollo y su integración a la sociedad en igualdad de condiciones.

Las cifras relacionadas con la alfabetización muestran una desventaja con respecto al género femenino; "dos terceras partes de analfabetos en el mundo son mujeres” (UNESCO, 2000).

En cuanto a la educación primaria y secundaria, a pesar de que el número de mujeres que asiste a la escuela se ha incrementado, sigue habiendo diferencias entre los porcentajes de ambos sexos. Más aún las tasas de deserción por diferentes motivos (labores de casa, cuidado de hermanos, embarazo precoz, etc.) es mucho mayor en mujeres.

Los países han planteado varias medidas y acciones para eliminar o disminuir esta desigualdad de la mujer en la educación: legislación, comité especial, publicaciones, foros, difusión en medios de comunicación, entre otros.

\section{EDUCACIÓN UNIVERSITARIA}

Si el acceso de la mujer a la educación básica y técnica fue complicado y tomó mucho tiempo en ser aceptado por la sociedad y la población masculina, la educación universitaria también presentó grandes obstáculos para el sexo femenino.

Alicia Italí Palermo (2013), presenta un excelente artículo titulado "El acceso de las mujeres a la educación universitaria". En el incluye una descripción del esfuerzo realizado por las mujeres y también por aquellas personas que trabajaron a favor de laigualdad deoportunidades educativas. Comienza citando una ilustrativa lista del Decreto de la Universidad de Bologna (1377), que a la letra dice:

Ya que la mujer es la razón primera del pecado, el arma del demonio, la causa de la expulsión del hombre del paraíso y de la destrucción de la antigua ley, y ya que, en consecuencia, hay que evitar todo comercio con ella, defendemos y prohibimos expresamente que se permita introducir una mujer, cual quiera que ella sea, aunque sea la más honesta, en esta universidad.

Relata que, en Roma la antigua, la mujer podía tener rudimentos de lectura, escritura y aritmética. Pero que, con la expansión del imperio, las mujeres patricias llegaron a obtener conocimientos de oratoria, historia y filosofía. Asimismo, que en el renacimiento, Magdalena Comedi Noé y María Pellegrina Amoretti obtuvieron el doctorado en Derecho en las universidades de Bologna y Padua respectivamente. En 1732, Laura Bassi obtiene el doctorado en Filosofía en la citada Universidad de Bologna.

También, menciona que algunas mujeres ejercieron docencia universitaria: María Delie Donne (obstetricia), María Caetama Agnesi (Física). Así también que Anna Manzolini estudiaba astronomía. Sostiene que la 
Universidad de Padua reservaba cinco vacantes para estudiantes mujeres. Formalmente, en el siglo XIX, se reglamentó en Italia el acceso de las mujeres a la Universidad.

Un hecho interesante, que menciona la autora, es que Dorotea Erxleben se recibió de doctora en medicina en 1754, en Alemania (Universidad de Halle), con la tesis escrita en latín "Examen de las causas que alejan a la mujer del estudio", lo que representa un excelente antecedente en la lucha de la mujer por la educación superior.

En otros países (Francia, Inglaterra, España) se inician movimientos a favor de la inclusión de la mujer en las universidades. Personajes importantes de estos países sostenían que "la mente no tiene sexo". Pero, aparecieron voces contrarias, entre ellas las de Rousseau y Phroudhon. Por ello, no es extraño que dos mujeres se graduaron como médico como si fueran varones: Miranda Stuart Barry (Ediburgo, 1812) y Enriqueta Faver Caben de Renau (Paris, 1971). Por esa época, la inglesa Mary Wollstonecraft, con su libro "Vindicación de los derechos de la mujer", se enfrenta a Rosseau, en defensa de la causa femenina.

Es en el siglo XIX cuando se comienza atender a las mujeres en las universidades (París, Zurich, Inglaterra, Italia, España, Bélgica, Dinamarca, Alemania, y Rusia).

Este impulso y acceso para las mujeres llega a América Latina, a fines del siglo XIX. La carrera que dio más acogida fue la de medicina. Gay (citado por Palermo), afirma que "el impulso a la medicina parecía natural en las mujeres, tan natural como la enseñanza, pues las esposas y madres eran en el siglo XIX, como lo habían sido siempre, las supervisoras de la salud y las enfermeras en el hogar".

En Estados Unidos, por su propia historia y cultura, en el siglo XIX, las mujeres eran más independientes e interesadas en las luchas sociales y entre ellas la lucha por la Educación. En 1835, Harriot Hunt estudió medicina en Boston en forma privada y rindió el examen de grado en forma libre, pues las mujeres no podían ingresar a la Universidad.

Palermo, afirma que en el año 1837 el Oberling College fue la primera institución universitaria que empezó a aceptar a mujeres, pero que el currículo era diferente para ambos sexos, y que la primera promoción femenina se graduó en 1841. La primera Escuela Normal para mujeres fue creada por Horace Mann en Massachussets en 1838, luego dichas escuelas se diseminaron a lo largo del país.

Más adelante, por iniciativa de personas educadas por la Iglesia, se crean Colleges independientes donde puedan asistir únicamente mujeres. Llegaron a crearse siete de ellos con gran prestigio, constituyéndose en filiales de siete universidades reunidas en la Ivy League. Se les llamó, entonces, Seven Sisters. En estas instituciones el currículo no discriminaba por sexo.

Las universidades privadas y estatales comenzaron a aceptar mujeres en la carrera de medicina (Utha, Iova, Baltimore, Yale, Cornell). La apertura de Estados Unidos hizo que mujeres de otros países llegaran a estudiar a sus universidades. Así, llegaron las hermanas inglesas, Elizabeth y Emily Blackwell; la canadiense Emily Harvard y la primera brasileña y la latinoamericana María Augusta Generoso Estrella. Esta última en 1881.

Para 1893, McCabe (citado por Gay) afirma que había 36,329 estudiantes mujeres en las instituciones de educación superior (11,718 en coeducación) y que para 1890 las mujeres representaban el 17\% de graduadas de Colleges $\mathrm{y}$ universidades estatales.

A medida que las mujeres lograban el acceso a la universidad, se abrió el debate sobre qué 
tipo de carreras eran las más "propias" para las mujeres. El área médica fue la que mostró mayor apertura. Sin embargo, esto no fue nada sencillo. Por ejemplo, la inglesa Elizabeth Blackwell, que se había recibido de médico en Estados Unidos, no pudo ejercer la profesión en Inglaterra dado que el parlamento inglés aprobó un Acta médica, en 1858, que restringía el campo de la medicina solo a los varones. Por ello, es que las mujeres tuvieron que luchar contra estas y otras discriminaciones a través de debates, reclamos públicos, apelaciones judiciales, viajes a otros países para estudiar medicina, etc.

Posteriormente, se les permitió realizar estudios de obstetricia, lo que representó una ventana para continuar avanzando. Otros países, además de Estados Unidos, permitieron los estudios de la mujer en el campo de la medicina: Suiza, Francia, Rusia y; posteriormente, Inglaterra. Esta apertura hacia la medicina es mayor cuando, a mediados del siglo XIX, se concluye que gran parte de la mortalidad infantil se debía al desconocimiento de las mujeres de las más elementales normas de higiene (Lemione, 1986, citado por Palermo).

En Europa, lalucha por los estudios universitarios de la mujer fue demandante, llegándose hasta los tribunales. Gay (citado por Palermo), sostiene que "en términos generales, fue el acceso a las universidades a finales del siglo XIX, lo que demostró ser la clave para la causa de la mujer, más que el acceso al voto".

Si bien, la mujer podía graduarse en una universidad, no podía ejercer. Esto era otro problema a resolver en medio de críticas de personajes reconocidos en el país. Proudhon fue muy duro pues sostuvo constantemente la inferioridad física, intelectual y moral de la mujer, ante lo cual Jenny d'Hericourt (graduada de médico en 1848 en la Universidad de París), replicó diciendo que es falso que la naturaleza hizo al hombre racional y a la mujer emocional.
En la Universidad de París se graduaron como médicos: Isabel Garret de Anderson (inglesa) en 1868 y en 1867 Nodezkka Suslova (rusa). Como farmacéutica se graduó Matilde Theussen (alemana) en 1864. Se dice que en 1882 estudiaban en la Universidad de París 32 mujeres, de las cuales 14 eran inglesas, 12 rusas y solo 6 eran francesas.

En la Facultad de Medicina de la Universidad de Zurich estudiaron 17 mujeres en 1870, la mayoría extranjeras, especialmente rusas.

La Universidad de San Petersburgo, en 1870, permitió el ingreso de mujeres a medicina, pero en 1881 cerró el acceso por temor del influjo del movimiento revolucionario en las estudiantes universitarias.

En Inglaterra, ante el impedimento a Elizabeth Blackwell de ejercer la profesión, le siguió un gran debate nacional. John Stuart Mills publicó en 1869, la obra "The subjection of woman" en la que sostenía que "lo que nosotros llamamos ahora la naturaleza de la mujer es eminentemente una cosa artificial, es el resultado de una represión forzada en algunas direcciones" (citado por Palermo). Asimismo, que "solo sabremos de las exactas diferencias entre hombres y mujeres cuando ambos sexos sean educados igualmente" (citado por Ungo).

En 1865, Joseph Payne había afirmado que "el espíritu propiamente no tiene sexo". En medio de estas argumentaciones, la Universidad de Edimburgo abrió las puertas a las mujeres en la carrera de medicina en 1868 -siendo su primera graduada Sofía Jex Blake. Posteriormente, en la misma carrera, permitieron el ingreso de mujeres a las universidades de Glasgow y Cambridge.

Sin embargo, no todo era color de rosa, la Universidad Senates no permitió que mujeres rindieran exámenes de ingreso y la Universidad de Oxford recién lo permitió a fines del siglo XIX. 
Más aún, hasta 1927 el número de ingresantes mujeres en Oxford estaba restringido.

En España, se recibió como Doctora en Filosofía y Letras Humanas (Universidad de Alcalá de Henares), en 1785, María Isidra Guzmán de la Cerda, autorizada por una Real Orden del Rey Carlos III. Se menciona el caso de Concepción Arenal, estudiante de Derecho, entre 1841 - 1846, como oyente en la Facultad de Derecho de la Universidad de Madrid, quien asistió a clases disfrazada de hombre.

María Elena Masseras se matriculó en medicina en la Universidad de Barcelona mediante un permiso especial del Rey Amadeo de Saboya (Real Orden del 2/9/1871) y en 1878 recibió su título de médico, siendo la primera médico en España.

En 1879, en la misma universidad, María Dolores Aleu Riera también se graduó de médico. Sin embargo, ninguna de las dos asistió a clases (régimen privado), solo rendían los exámenes en fechas predeterminadas. Se cuenta que María Elena Masseras, asistió a un curso acompañada por su hermano que también estudiaba medicina, Posteriormente, viajaron a Madrid para estudiar el Doctorado.

María Elena, desistió por los trámites y dificultades en el proceso de admisión; en cambio, Dolores si llegó a estudiar y se convirtió en la primera doctora en medicina en España.

En la Universidad de Barcelona, también se tituló de médico Martina Castells Ballespi y se doctoró en Madrid en 1882. Las primeras licenciadas en Farmacia fueron: Eloísa y María Dolores Figueroa Marti.

En 1888, la Real Orden del ll de junio permitió los estudios de las mujeres como "alumnas de enseñanza privada”. Bajo esta autorización, Teresa de Andrés Hernández se recibió de Licenciada (1889) y Ángela Carrafa de Nava, de Doctora (1892). Ambas, en Filosofía y Letras.

Posteriormente, hasta 1910, para poder estudiar en la universidad, las mujeres tenían que solicitar permiso a la Dirección General de Instrucción Pública. La respuesta era lenta y podía tardar años. Por este motivo, se afirma que en el siglo XIX fueron pocas las mujeres en las universidades españolas.

El 8 de marzo de 1910 el Ministerio de Instrucción Pública, a cargo del Conde de Romanones, publica una orden que permite la matrícula de mujeres en toda Institución Educativa, eliminándose, de esta manera, los permisos requeridos anteriormente.

Es así que se gradúan como primera Licenciada María Sordé Xipell (1914) y de primera Doctora Catalina de Sena Vives Pieras (1917). Ambas en ciencias. La primera Doctora en Farmacia (1918) fue Zoe Rosinach Pedrol. La primera Licenciada en Derecho (1922) fue María Asunción Chrivella Marín y la primera Doctora fue Carmen Cuesta del Muro. La primera Ingeniera Industrial (1929) fue Pilar Careaga Basade y Matilde Ucelay Maortúa, la primera Arquitecta (1936).

En Bélgica y Dinamarca las universidades abrieron sus puestos a las mujeres alrededor de 1875 . Años previos, podían inscribirse en las universidades finlandesas, mediante un permiso especial que se mantuvo hasta 1907.

En Alemania, la Universidad de Gottingen recién empieza a aceptar mujeres a partir de 1884, de allí egresan las rusas: Sophia Kevaleskaia (Matemática) y Julia Lermontova (Química). Los títulos eran "honorarios" pues no les permitía ejercer legalmente la profesión.

Kovaleskaia fue la primera doctora en una universidad alemana y la segunda mujer en recibir un premio de las Ciencias Francesas. 
Palermo, sostiene que Alemania fue el único país europeo que permitió a las mujeres estudios en otras ramas que no fueran las del área médica.

Así, en 1898 se recibió de Doctora en Matemática la Condesa María Von Liden en la Universidad de Berlín y, desde 1898 la Universidad de Giessen abre sus puertas a las mujeres en la Facultad de Filosofía (antes lo había hecho la Universidad de Yena).

En Noruega, la admisión de mujeres empieza en 1884. En Australia, se abrió una universidad femenina para estudiar medicina. En Viena, hubo gran discusión para permitir el ingreso de la mujer a la universidad y, en especial, a la Facultad de Medicina. No obstante, en la Universidad de Kaskof les era permitido estudiar la carrera de Medicina. La primera doctora en la Facultad de Filosofía fue la Condesa de Warsteuslebur (1899).

La carrera de Derecho fue la más reacia a admitir mujeres. Por ejemplo, la Universidad de Viena aprobó el ingreso de mujeres recién en 1900, pero los varones recibieron con insultos a las mujeres que se inscribieron (López, 1901, citado por Palermo). Asimismo, a la suiza Emilie Kemfin-Spyti matriculada en Derecho en la Universidad de Zurich, se le negó su licenciatura. Viajó a Estados Unidos donde creó el First Law College y a su regreso a Zurich se le vuelve a negar el título. La primera abogada se recibe en Estados Unidos en 1869 (20 años después de que lo hizo una médico) en la Universidad de Iowa.

El proceso de afirmación de la mujer en Derecho y en otras carreras tuvo que vencer las siguientes trabas:

, Ingreso formal a la universidad.

> Ejercicio legal de la profesión

> Consolidación en la profesión.

\section{LAS UNIVERSITARIAS EN AMÉRICA LATINA}

De acuerdo a Palermo el acceso de las mujeres a las universidades se da a partir de la década de 1880; principalmente, en los siguientes países: Brasil, México, Chile, Cuba y Argentina. Medicina, continúa siendo la carrera preferida.

En diferentes países, en el siglo XIX, los diarios participaron activamente en la promoción de los derechos de la mujer y otros periódicos y revistas con tinte feminista colaboraron en que las mujeres lleguen a realizar estudios universitarios.

Este logro ocurrió casi medio siglo después del acceso femenino a la universidad en Estados Unidos. La primera graduada pareciera que se dio en México y fue Matilde Montoya (1887), quien obtuvo su título de Médico Cirujano después de enfrentar grandes obstáculos.

Zenaida Ucounkoff se tituló de Médico, pero no contaba con el registro de su ejercicio profesional. La primera latinoamericana titulada de médico fue la brasileña Augusta Generoso Estrella; pero lo hizo en Estados Unidos. Otros connacionales de Augusta que estudiaron también medicina en Estados Unidos fueron: Josefa Águeda Felisbella y Mercedes de Oliverira. En el mismo Brasil, Rita López se matriculó en medicina en 1887 en la Universidad de San Salvador de Bahía. En Río de Janeiro se recibieron de médicos, en 1888 y 1889 respectivamente, Hermelinda de Vasconcellos y Antonieta César Díaz.

En Cuba, Mercedes Riva Pinos, se licencia en 1885 de la Facultad de Filosofía y Letras y obtuvo el doctorado en 1887. Al año siguiente (1888), Francisca de Rojas Cabaret es declarada Licenciada en Derecho Civil y Canónico. Ese mismo año obtuvo la Licenciatura en Ciencias (Físico - Matemáticas), Digna América del Sol y Gallardo quien ingresó a la universidad a los 15 años de edad. En 1888, Laura Martínez Carbajal 
y del Camino López se graduó de Licenciada en Ciencias Físico - Matemáticas, carrera que no ejerció. En 1890, obtiene la Licenciatura en Medicina y se especializó en Oftalmología y trabajó 20 años al lado de su esposo con la misma especialidad. Años más tarde (1898), se licencia de Médico, María Teresa Mederos y Rodríguez. La primera mujer matriculada en Farmacia fue María de la Asunción Menéndez de Luarca Díaz.

En Guatemala, en los años 20 del siglo pasado, las mujeres iniciaron su ingreso a la Pontificia Universidad de San Carlos. Años previos, se les permitía realizar estudios de comadronas o parteras en la Escuela Anexa de Comadronas de la Facultad de Medicina (1883), o graduarse de Maestras de Educación Primaria (18714 - 1920).

Un caso singular es el de Olimpia Altuve, quien en 1919 se convirtió en la primera mujer graduada universitaria de Centro América, la primera mujer egresada de la Facultad de Ciencias Naturales y Farmacia con el título de Farmacéutico y la primera egresada de la Universidad Nacional. Sin embargo, no pudo ejercer debido a limitaciones legales hasta que fueron eliminadas y, por fin, se le reconoció como Química Bióloga.

En 1927, Luz Castillo Ordaz obtuvo el título de Abogado y Notario; y en 1947, Francisca Fernández Hall obtuvo el título de Ingeniera Civil. En 1942, María Isabel Escobar Quintana se graduó de Médico Cirujano; en 1943, Graciela Quan Valenzuela es la primera mujer en obtener la licenciatura en leyes pero, al igual que en otros casos, no pudo ejercer su profesión.

En Chile, en 1877, el Ministro de Instrucción Pública, Miguel Amunátegui, dictó el decreto mediante el cual se permitía el ingreso de las mujeres a la universidad. No obstante, en los primeros años de la década habían ingresado algunas mujeres a la universidad (medicina): Eloiza Díaz Insunza y Ernestina Pérez, siendo Eloiza la primera mujer en América del Sur en obtener el títulode Doctora en Medicina y Cirugía
(1887) en una universidad latinoamericana. Matilde Throup es la primera Abogada (1892).

En Argentina, a pesar de que su Constitución de 1853 reconocía igualdad de derechos para ambos sexos, la educación femenina generó un gran debate desde mucho antes, e.g. 1810. Manual Belgrano, sostenía que "la educación de la mujer era piedra fundamental de una nueva nación" (citado por Palermo).

Pero, fue Sarmiento quién bregó fuertemente por la educación de la mujer, basado en la información que tenía de lo que ocurría en otros países y en sus propias creencias. Por este motivo, cuando se encontraba en la Presidencia, el Congreso de la Nación autorizó, en 1869, la creación de Escuelas Normales, inaugurándose la primera en Paraná en 1870. Para facilitar el funcionamiento de las escuelas se contrató a 65 profesoras norteamericanas, de diferentes Estados, en el periodo transcurrido entre 1869 a 1890.

Elida Passo se recibió de Farmacéutica en 1885 y, posteriormente, logró estudiar medicina por un recurso judicial interpuesto ya que se le había negado la inscripción a la carrera. Passo fue la primera mujer inscrita en una carrera universitaria y pudo ser la primera Médico, pero falleció antes de concluir sus estudios.

En 1824, la francesa Verónica Pascal fue la primera mujer en Argentina de obtener el diploma de Obstetra; y en 1888, Celina de Duval, otra francesa, revalidó su título de Odontóloga en la Escuela de Medicina de la Universidad de Córdoba, el cual había recibido el mismo año en la Universidad de Montevideo.

En 1889, Ángela Zuluaga de Bourouconos solicitó rendir exámenes de medicina a la respectiva Facultad de la Universidad de Buenos Aires, acompañando un certificado de un reconocido médico que acreditaba las lecciones recibidas por la solicitante de su parte y del esposo de Ángela (Dentista). 
En 1892, se crea la Escuela de Odontología en la Facultad de Ciencias Médicas. Allí se inscribieron: Cidanelia Gónzalez (recibió su título en 1896), Fanny Bitz (rusa, recibió el título en 1896) y las hermanas Petrona y Josefina Pecotche.

En el caso de la carrera de Farmacia, tres mujeres se recibieron de Farmacéuticas y aspiraron luego a la carrera de Medicina: Elida Passo, antes mencionada, Julieta Lauteri y Fanny Bache. Las dos últimas se recibieron de médicos, años después.

La carrera de Medicina fue iniciada por Élida Passo mediante autorización judicial. Pero, la primera Médico en Argentina fue Cecilia Grierson. La segunda fue Petrona Eyle en 1891, en una universidad de Zurich. Su título fue revalidado en 1893 por la Facultad de Medicina de la Universidad de Buenos Aires. En 1892, en la misma universidad se recibió de Médico Elvira Rawson Guiñasu.

Con la creación de la Facultad de Filosofía y Letras las mujeres pudieron reorientar sus estudios a otras carreras más relacionadas con "la naturaleza femenina" y, además, porque estas carreras "no abren camino para la fortuna... y les presenta una escasa perspectiva de lucro" (Palermo).

En Colombia, recién se permite el acceso de las mujeres a la universidad en 1934 mediante un proyecto de ley presentando al Congreso de la República. Este hecho generó una gran discusión, como sucedió en otros países.

El proyecto fue defendido por Jorge Eliécer Gaitán y atacado por Germán Arciniegas. (Ramírez, M. H). Gerda Westendorp fue admitida, en 1935, en la carrera de Medicina en ese país, pero, fue Gabriela Peláez quien ingresó en 1936, se convirtió en la primera mujer graduada de Abogada en la universidad.
En Uruguay, Paulina Luisa es la primera mujer médico (estudió medicina en Montevideo) y es la primera mujer en ocupar una cátedra universitaria. Su hermana Inés fue Doctora en Medicina y la otra hermana, Clotilde, fue la primera mujer que se recibió de Abogada en ese país.

\section{LAS PERUANAS UNIVERSITARIAS}

María Trinidad Enriquez es considerada como la primera mujer en ingresar a una universidad peruana. En 1875, ingresó a la Facultad de Jurisprudencia de la Universidad de Cusco (en 1874 recibió la autorización presidencial para hacerlo) pero no llegó a graduarse debido a las dificultades que encontró en su camino.

$\mathrm{Al}$ concluir sus estudios tuvo que recurrir al Congreso para tramitar sus grados académicos. Debido a la Guerra del Pacifico, su solicitud no fue tramitada, sino después de la misma y fue derivada a la Corte Superior de Lima, que le negó el bachillerato. Al respecto, es interesante conocer el argumento del fiscal Ricardo Espinoza sobre la solicitud de María Trinidad Enriquez, en 1891:

Las profesiones que exijan un notable vigor físico, un gran poder intelectual o una voluntad firme y enérgica, modifican profundamente la naturaleza de la mujer y casi la convierten en hombre, privándola de las cualidades especiales que provocan la unión de los dos sexos, que forman la familia y hacen el encanto del hogar, base fundamental de la sociedad (citado por Valladares Chamorro, 2012).

Mientras seguía su lucha en los tribunales por lograr sus grados y autorización para ejercer, falleció un 20 de abril de 1891.

Palermo hace referencia a Margarita Práxedes Muñoz, quien en 1895 se recibió de Médico. Fue criada por sus abuelos al quedar huérfana. La familia, de buena educación y grandes relaciones, 
incentivó en ella afanes intelectuales. Se graduó en Ciencias Naturales en Lima (1890) e ingresó a estudiar Derecho en la Universidad San Marcos pero dejó los estudios, presumiblemente, por dificultades que tenían las mujeres para estudiar Derecho (Palermo). Viajó a Chile en donde se tituló de Médico. Allí ejerció su profesión, luego lo hizo en Argentina.

En el Perú, al igual que en otros países, las mujeres no tenían al inicio oportunidades de educación; especialmente, de Educación Universitaria. Las inquietudes educativas de desarrollo chocaban con la actitud de los varones, la legislación y las costumbres sociales. Para poder realizar estudios superiores debían ser autorizadas por el Estado. Por ejemplo, Esther Festini, fue autorizada el 16 de diciembre de 1899 para ingresar a estudiar en la Universidad y María Luisa Molinares el 29 noviembre de 1902.

Laura Esther Rodríguez Dulanto es la primera Médico peruana (1900). Otras tituladas son: Eudoxia Pauta, Herminia Gonzales y Valeria Von Westernhagen Rumann. Asimismo, la Dra. Leopoldina Gaviño Matías (esposa de Enrique Guzmán y Valle), Esther Festini de Ramos Ocampo (primera Doctora en Letras, 1904), María Elvira Rodríguez Lorente (sobrina de Sebastián Lorente) y la Pedagoga María Luisa Molinares de Reátegui. También se menciona en este grupo pionero a Julia Amanda Iglesias, Leonor Incháustegui, Dorita Isolina Soto, Feliza Faura Bedoya, Mercedes Patrón y Ferry, Agusta Ugarte y Cristina Patrón y Ferry.

Recién en 1908, el presidente Augusto B. Leguía, mediante Ley № 081 (7 de noviembre), firma la propuesta del Congreso para que "las mujeres que reúnan los requisitos que la ley exige para el ingreso a las universidades de la República, sean matriculadas en ellas cuando así lo soliciten, pudiendo optar los grados académicos y ejercer la profesión a que se dediquen" (Valladares Chamorro, 2011).
Palermo, afirma que en 1917 un visitante peruano a la Escuela de Odontología de la Facultad de Ciencias Médicas de Buenos Aires (Argentina) quedó muy impresionado por el porcentaje de mujeres que estudiaba en dicha Escuela.

Lo cierto es que el ingreso de las mujeres a las universidades comienza a ser significativo a partir de la segunda mitad del siglo XX. Datos de la Asamblea Nacional de Rectores (ANR), Censo Universitario 2010, muestran los porcentajes de ingresantes, según sexo para ese año: varones = $50.6 \%$ y mujeres $=49.4 \%$.

Una visión más precisa del número de estudiantes de pregrado en todo el Perú aparece a continuación:

\section{Tabla 1}

Estudiantes de Pregrado en Universidades Peruanas según tipo de universidad y sexo

\begin{tabular}{lccc}
\hline UNIVERSIDAD & $\mathrm{H}$ & $\mathrm{M}$ & Total \\
\hline Privada & 226,052 & 247,743 & 473,795 \\
& $(48 \%)$ & $(52 \%)$ & $(100 \%)$ \\
Pública & 174,093 & 135,082 & 309,175 \\
& $(56 \%)$ & $(44 \%)$ & $(100 \%)$ \\
\hline Total & 400,145 & 382,825 & 782,970 \\
& $(51 \%)$ & $(49 \%)$ & $(100 \%)$ \\
\hline
\end{tabular}

Fuente: ANR, Resumen Estadístico Universitario 2010.

De acuerdo a la tabla 1 , las mujeres encuentran mayor acogida en universidades privadas. En relación a todo el país, en el 2010, el número de varones y mujeres que asistieron a la universidad es similar. Esto implica el gran avance realizado por las mujeres para hacer uso de su derecho a la educación universitaria.

Sin embargo, todavía hay mucho camino por transitar en otros aspectos. Por ejemplo, del portal Web de la ANR, haciendo uso de la relación de autoridades de las universidades formales, con autorización definitiva en el Perú, se ha construido la tabla siguiente. 
Tabla 2

Autoridades Universitarias según tipo de universidad, sexo

\begin{tabular}{lcccc}
\hline \multirow{2}{*}{ UNIVERSIDADES } & \multicolumn{2}{c}{ RECTOR } & \multicolumn{2}{c}{ VICERRECTOR } \\
& H & M & H & M \\
\hline Públicas & 29 & 1 & 49 & 11 \\
(Total) & \multicolumn{2}{c}{$(30)$} & \multicolumn{2}{c}{$(60)$} \\
\hline Privadas & 42 & 4 & 58 & 12 \\
(Total) & \multicolumn{2}{c}{$(46)$} & \multicolumn{2}{c}{$(70)$} \\
\hline Total & 71 & \multicolumn{2}{c}{107} & 23 \\
(Total) & \multicolumn{2}{c}{ (76) } & \multicolumn{2}{c}{$(130)$} \\
\hline
\end{tabular}

Fuente: Página Web - ANR/ Directorio 2013.

La tabla 2 muestra que de 76 Rectores solo 5 $(6.6 \%)$ son mujeres, mientras que en el caso de los Vicerrectores hay 23 de 130 (17.7\%). Llama la atención que en Universidades Públicas exista solo 1 mujer de 30 Rectores (3.3\%). El número de mujeres Vicerrectoras en Universidades Públicas y Privadas es casi igual; pero los porcentajes son diferentes: públicas $(11-18.3 \%)$, privadas $(17.7 \%)$.

\section{A MANERA DE RESUMEN}

Las mujeres han realizado una gran batalla por el logro de sus derechos, especialmente el de la educación. Desde la alfabetización para su inserción en la vida cívica del país, a la Educación Básica para el logro de sus derechos civiles, a la educación técnica para favorecer sus ingresos y el desarrollo del sistema productivo, hasta la educación superior universitaria para el logro pleno de sus derechos ciudadanos. Han tenido que enfrentarse al "poder del hombre" en sociedades que no las respetaban en su integridad, pues le asignaban únicamente roles tradicionales.

El trajín por acceder a la universidad ha sido similar en todos los países: (1) oposición, (2) permisos (Rey, Congreso), (3) apertura en ciertas carreras, (4) permisos limitados para ejercicio de la profesión, (5) oposición interna (hostilidad de los docentes / ausencia de servicios para mujeres, títulos y grados con denominación masculina), (6) oposición externa (temores de la sociedad, desconfianza, inseguridad).

La educación formal, la educación universitaria, es una construcción social, política, económica y cultural que no debe excluir a las personas por cuestión de género. El avance de la mujer en la universidad, actualmente, ha sido grande en el Perú y en otras partes del mundo. La sociedad, en su conjunto, debe sentirse orgullosa de estos logros y debe, además, continuar transitando por el camino de la igualdad de género en la educación superior y en todos los contextos, completando los vacíos que aún subsisten; especialmente en el ejercicio profesional de la mujer. 


\section{REFERENCIAS}

Archivo Nacional de Chile. Decreto Amunátegui. Mujeres a la Universidad. Recuperado de http://www. dibam.d:_8182/vistas_publicas/publicContenido/ ContenidoPublicDetalle. aspx?folio $=6986$

Asamblea Nacional de Rectores, Dirección General de Planificación Universitaria. (2012). Resumen Estadístico Universitario. Lima, Perú: Autor.

Barroyo, A. P. Una historia para contar: mujeres pioneras en al educación superior, primera mitad del siglo XX. Revista Mujeres y Universidad, l (l). Recuperado de http:// digi.usac.edu.gt/iumusac/sistemas/docs/Revista2005. pdf

Flecha García, C. (1996). Las primeras universitarias en España. Madrid, España: Narcea.

Marchante Castellanos, P., y Merchán Gonzalez, F. Las primeras estudiantes universitarias en Cuba. Recuperado de http://www.bvs.sld.cu/revistas/far/vol46_1_12/ farl4ll2.htm

Palermo, A. I. (2006). El acceso de las mujeres a la educación universitaria. Recuperado de http://www. scielo.org.ar/scielo.php?script=sci_arttxe\&pid $=$ S1669-32482006000200002

Peña, D. (15 de diciembre de 2012). Las primeras mujeres universitarias en España. [Mensaje en un blog]. Recuperado de http:/ginemartin.blogspot. com/2012/12/las-primeras-mujeres -universitarias-en. html

Ramírez, M. H. La primera mujer entra a la universidad. Recuperado de http://colombialink.com/01_index historia/07_otros_ hechos_históricos/0220_primera_ mujer_universidad.html
Silva Manzón, A. (2005). La perspectiva de género en el curriculum universitario. Revista Mujeres y Universidad, l(1). Recuperado de http://digi.usac.edu.gt/iumusac/ sistemas/docs/Revista2005.pdf

UNESCO (1997). Educación de la mujer. Recuperado de http://unesco.org/edcuation/elie/confitea//pdf/4a_ spam. pdf

UNESCO (2000). Educación y capacitación de la mujer. Recuperado de http://www.un.org/spanish/ conferences/Beijing/fs2.htm.

Ungo, U. A. (2007). Las mujeres y la educación en América Latina: una aproximación a los dilemas y desafíos. Revista Venezolana de Estudios de la Mujer, 12(8). Recuperado de http://webcache.googleusercontent. com/search? q=cache:fGJRIL2CUF4J:www. scielo.org.ve/scielo.php\%3fpid\%3DS1316$37012007000100011 \% 26$ script\%3Dsci_arttext+educ acion $+d e+l a+$ mujer $+e n+e l+\operatorname{siglo}+x x \& c d=64 \&$ hl =es $419 \& \mathrm{ct}=\mathrm{clnk} \& \mathrm{gl}=\mathrm{pe}$

Valladares Chamorro, O. R. (27 de julio de 2011). Cien años de mujeres universitarias en el Perú. 1908-2008. [Mensaje en un blog]. Recuperado de http://odalisdelima. wordpress.com/2011/07/27/cin-anos-de-mujresuniversitarias-en-el-peru-1908-2008-31

Valladares Chamorro, O. R. (2012). La incursión de las mujeres a los estudios universitarios en el Perú: 1875 - 1908. Recuperado de http://e-revistas.uc3m.es/index.php/ cian/article/view/1544/758

Vera de Flachs, M. C. (2005). Mujeres Latinoamericanas: su inserción en los estudios superiores y en el campo de la investigación científica. Rhec, (8), 49-76. 